\section{HIV/AIDS: Emerging threat to cardiovascular health in sub-Saharan Africa}

To the Editor: Public health in sub-Saharan Africa (SSA) is at a crossroads owing to an increasing non-communicable disease burden, with a sizeable contribution from cardiovascular disease (CVD). CVD is the top cause of death globally, ${ }^{[1]}$ and an estimated $80 \%$ of CVD-related deaths occur in low- to middle-income countries, including South Africa (SA). ${ }^{[2]}$ Although epidemiological transition is an important factor contributing to the rise in cardiovascular risk factors and $\mathrm{CVD},{ }^{[3]}$ the emerging interaction between communicable diseases such as HIV/AIDS and CVD should not be neglected. HIV infection is now recognised as an independent cardiovascular risk factor; however, the data are mainly from developed countries. ${ }^{[4,5]}$

The paucity of studies investigating this question in SSA, where demographic and socioeconomic realities, HIV strains and antiretroviral (ARV) guidelines are different, is of great concern. In high-income countries, HIV-related CVD manifests as coronary heart disease; ${ }^{[4]}$ conversely, the incidence of coronary heart disease is believed to be relatively low in HIV-infected SSA populations. ${ }^{[6]}$ Most reports cite pulmonary hypertension, cardiomyopathy and tuberculous pericarditis as the predominant HIV-related cardiovascular conditions. ${ }^{[6]}$ A recent cross-sectional study in Soweto, SA, showed that only $2.4 \%$ of patients presenting with coronary heart disease for the first time were HIV-positive. ${ }^{[7]}$ We believe that these and other findings may not represent the full picture or future trends of HIVrelated CVD in SSA. In recent years, data have started to emerge pointing to a high incidence of proatherogenic cardiovascular risk factors in HIV-infected participants, including hypertension, obesity, dyslipidaemia and endothelial activation. ${ }^{[5,8,9]}$ Unfortunately the vast majority of these studies are observational, cross-sectional or hospital based, with no longitudinal follow-up. This hampers thorough assessment of the incidence and nature of cardiovascular risk and disease trajectories in people living with HIV/AIDS. Furthermore, HIV infection is inextricably linked with antiretroviral therapy (ART), another putative cardiovascular risk factor. It is well established that several ARV drugs can result in increased cardiovascular risk; ${ }^{[10]}$ however, for as long as this possible link is not investigated in our context, serious future cardiovascular health challenges may arise, particularly in view of the massive ART roll-out programmes initiated in recent years. ${ }^{[6]}$

In conclusion, we believe there is a real possibility that HIV infection and ART are emerging driving forces behind the rapidly increasing cardiovascular risk factor and CVD rates in SSA. If the lack of population studies investigating this question persists, public health services may be even less prepared for the multiple burden of disease facing the continent.

Hans Strijdom, Patrick De Boever, Tim Nawrot and Nandu Goswami are members of the EndoAfrica research consortium. The EndoAfrica study is investigating the effects of HIV-infection and ARV treatment on cardiovascular health and endothelial function in SA populations.

\section{Hans Strijdom}

Cardiovascular Research Group, Division of Medical Physiology, Faculty of Medicine and Health Sciences, Stellenbosch University, Tygerberg, Cape Town, South Africa jgstr@sun.ac.za

\section{Patrick De Boever}

Environmental Risk and Health Unit, Flemish Institute for Technological Research (VITO), Belgium; Centre for Environmental Studies, Hasselt University, Belgium

\section{Tim S Nawrot}

Centre for Environmental Studies, Hasselt University, Belgium; Department of Public Health and Primary Care, Leuven University, Belgium

\section{Nandu Goswami}

Gravitational Physiology and Medicine Research Unit, Institute of Physiology, Medical University of Graz, Austria

1. Institute for Health Metrics and Evaluation (IHME), GBD Compare Seattle, WA IHME University of Washington, 2013. http://vizhub.healthdata.org/gbd-compare (accessed 8 February 2015).

2. Yusuf S, Rangarajan S, Teo K, et al. Cardiovascular risk and events in 17 low-, middle-, and highincome countries. N Engl J Med 2014;371(9):818-827. DOI:10.1056/NEJMoa1311890

3. Muna WF. Comprehensive strategies for the prevention and control of diabetes and cardiovascular disease in Africa: Future directions. Prog Cardiovasc Dis 2013;56(3):363-366. DOI:10.1016/ pcad.2013.10.012

4. Zanni MV, Schouten J, Grinspoon SK, Reiss P. Risk of coronary heart disease in patients with HIV infection. Nat Rev Cardiol 2014;11(12):728-741. DOI:10.1038/nrcardio.2014.167

Bloomfield GS, Hogan JW, Keter A, et al. Hypertension and obesity as cardiovascular risk factors among HIV seropositive patients in Western Kenya. PLoS One 2011;6(7):e22288. DOI:10.1371/ journal.pone.0022288

6. Mayosi BM, Flisher AJ, Lalloo UG, Sitas F, Tollman SM, Bradshaw D. The burden of non-communicable diseases in South Africa. Lancet 2009;374(9693):934-947. DOI:10.1016/S0140-6736(09)61087-4

. Sliwa K, Carrington MJ, Becker A, Thienemann F, Ntsekhe M, Stewart S. Contribution of the human immunodeficiency virus/acquired immunodeficiency syndrome epidemic to de novo presentations immunodeficiency virus/acquired immunodeficiency syndrome epidemic to de novo presentations
of heart disease in the Heart of Soweto Study cohort. Eur Heart J 2012;33(7):866-874. DOI:10.1093 of heart disease in

8. Julius H, Basu D, Ricci E, et al. The burden of metabolic diseases amongst HIV positive patients on HAART attending the Johannesburg Hospital. Curr HIV Res 2011;9(4):247-252 DOI: $10.2174 / 157016211796320360$

9. Fourie CM, Schutte AE, Smith W, Kruger A, van Rooyen JM. Endothelial activation and cardiometabolic profiles of treated and never-treated HIV infected Africans. Atherosclerosis 2015;240(1):154-160. DOI:10.1016/j.atherosclerosis.2015.03.015

10. Currier JS, Lundgren JD, Carr A, et al. Epidemiological evidence for cardiovascular disease in HIV-infected patients and relationship to highly active antiretroviral therapy. Circulation 2008:118(2):e29-e35.DOI:10.1161/CIRCULATIONAHA.107.189624

S Afr Med J 2016;106(6):537. DOI:10.7196/SAMJ.2016v106i6.10721 